Int Surg 2014;99:407-409

DOI: 10.9738/INTSURG-D-13-00072.1

\title{
Giant Spermatic Cord Liposarcoma
}

\author{
Ugo Grossi ${ }^{1}$, Antonio Crucitti ${ }^{1}$, Francesco Pierconti ${ }^{2}$ \\ ${ }^{1}$ Department of Surgery and ${ }^{2}$ Department of Pathology, Catholic University of the Sacred Heart, Largo A. \\ Gemelli 8 - 00168 Rome
}

\begin{abstract}
We report a case of giant spermatic cord liposarcoma (SCL) in an 81-year-old patient, presenting with a huge scrotal mass that reached up to the knee joint. SCL is a rare tumor, and about 200 cases have been reported in the literature so far. Although $20 \%$ of liposarcomas arise in the retroperitoneum, only $0.1 \%$ present as incidental inguinal hernias. The occasional presence of myxoid stroma in well-differentiated liposarcomas can lead to confusion with myxoid sarcoma subtypes. Correct diagnosis is critical and reflects remarkable differences in behavior and therapeutic choices.
\end{abstract}

Key words: Liposarcoma - Spermatic cord - Scrotal mass

$S^{\mathrm{p}}$ permatic cord liposarcoma (SCL) is a very rare tumor, and about 200 cases have been described in the literature up to date. Clinically, these tumors can sometimes mimic the common scrotal masses such as hernias, testicular cancer, and hydrocele. Their histologic heterogeneity reflects remarkable differences in both prognosis and treatment. We report a case of SCL in an 81-year-old patient presenting with a huge scrotal mass.

\section{Case Report}

An 81-year-old Caucasian man was admitted to the hospital with a giant, painless, scrotal mass that started growing 4 years before presentation, showing a progressively increasing volume over time. The patient had a previous history of laryngeal carcinoma treated with curative surgery. Two weeks prior to hospital admission, he developed acute urinary retention due to a severe concealed penis, requiring prompt bladder catheterization. Physical examination showed a greatly enlarged scrotum due to a huge lobulated mass extending to the knee level (Fig. 1). The scrotal skin appeared highly stretched, but otherwise normal.

Ultrasound examination revealed a hyperechogenic and inhomogeneous mass, along with the superior and lateral dislocation of both testes. Contrasted computed tomography (CT) documented fat attenuation intermixed with soft-tissue attenuation nodules along with no evidence of regional lymphadenopathies. The patient underwent complete excision of the mass (Fig. 2) with high dissection of the spermatic cord and radical right orchiectomy (weight of the specimen, $5130 \mathrm{~g}$; size, $28 \mathrm{~cm} \times 30 \mathrm{~cm} \times 15 \mathrm{~cm}$ ). On macroscopic examination, the tumor presented multiple yellowish-white rubbery nodules (Fig. 3). Histology re- 


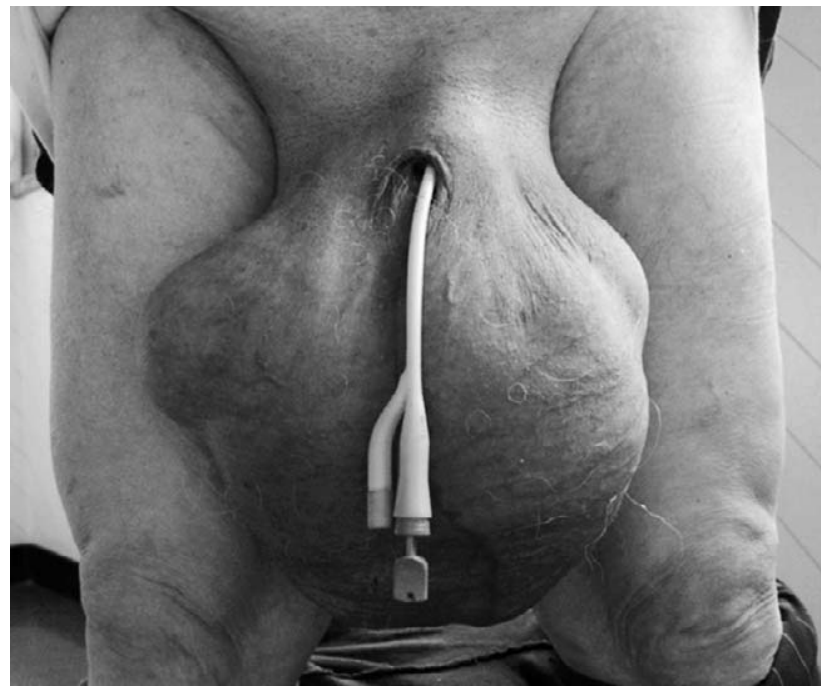

Fig. 1 Huge lobulated scrotal mass extending to the knee level with associated severe concealed penis. A Foley's catheter has been inserted.

vealed a well-differentiated liposarcoma with mixoid degeneration arising from the right spermatic cord and in continuity with the testis. The patient did not undergo any adjuvant radiotherapy or chemotherapy and is currently disease free at 5year follow-up after surgical resection.

\section{Discussion}

Liposarcomas account for 3\% to $7 \%$ of malignant paratesticular tumors, presenting as incidental inguinal hernias in only $0.1 \%$ of cases. Lesauvage first reported SCL in $1845,{ }^{1}$ and about 200 cases have been described in the world literature so far.

At gross examination, SCLs appear as heterogeneous, multilobulated, well-defined, lipomatous masses, with variable consistency on palpation. The most common CT finding of well-differentiated SCL is a large mass of fat attenuation $(>75 \%$ of the lesion) intermixed with nonlipomatous thick septa or soft-tissue attenuation nodules. ${ }^{2}$

The differential diagnosis includes inguinoscrotal hernia, testicular cancer, and hydrocele. Giant inguinoscrotal hernias are defined to extend below the midpoint of the inner thigh in the standing position. ${ }^{3}$ Omentum and small bowel are commonly found in the hernia sac. Care should be taken to identify the potential complications, which include intestinal occlusion, incarceration, and strangulation of the contents.

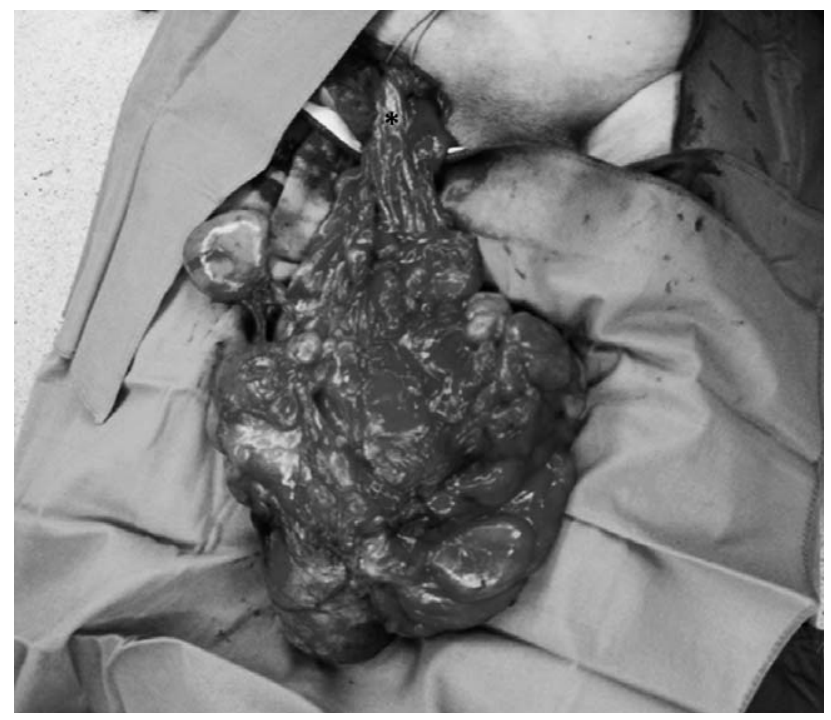

Fig. 2 The mass has been totally pulled out from the scrotal sac. The spermatic cord (asterisk) has been encircled with blue vascular tape.

Testicular cancer affects males mainly in their young adult years. Germ cell tumors represent the most common histotype, occurring in $95 \%$ of cases. Up to $90 \%$ of patients present with a painless scrotal mass, with a history of trauma or bruising and hydrocele or hernia in less than $10 \%$ of cases. ${ }^{4}$

Hydrocele is a fluid collection that occurs in the inguinal canal as a result of congenital nonclosure of the vaginal process. Giant hydrocele has been defined as having more than $1000 \mathrm{ml}$ of contents. ${ }^{5}$ Groin swelling and a fluctuant palpable mass are typically observed at physical examination.

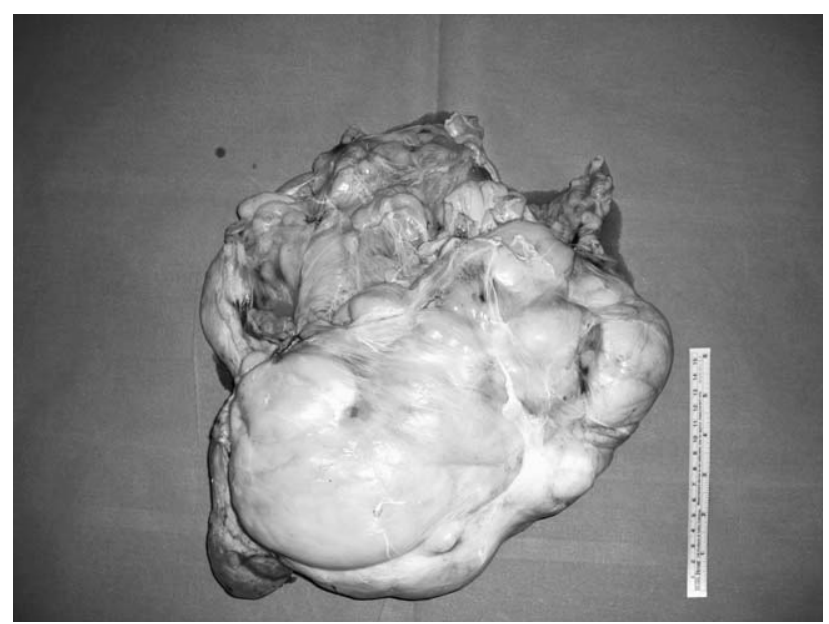

Fig. 3 The tumor presents multiple yellowish-white nodules with rubbery consistency. 
Liposarcomas are histologically classified into 4 subtypes (well-differentiated, dedifferentiated, myxoid, and pleomorphic) based on natural history, morphologic features, and cytogenetic alterations. ${ }^{6}$ The so-called mixed-type refers to a small group of liposarcomas with combined features of 2 or more subtypes. Occasionally, well-differentiated liposarcomas contain myxoid stroma and can be misinterpreted as myxoid liposarcomas. Correct diagnosis is critical in such cases owing to remarkable differences in both prognosis and treatment. Indeed, unlike the well-differentiated subtype, myxoid liposarcoma has a high risk of metastasis and is highly sensitive to chemotherapy and radiation. In a recent 14 -year case series of 56 liposarcomas with prominent myxoid stroma, $22(39 \%)$ were the well-differentiated subtype, and $11(20 \%)$ arose in the spermatic cord. $^{7}$

Radical orchiectomy with wide local excision is the mainstay of treatment strategies. Long-term follow-up is mandatory due to the high recurrence rate, which reached up to $55 \%$ in a 20 -year case series of spermatic cord sarcomas. ${ }^{8}$ The benefit of adjuvant radiotherapy in reducing local recurrences is not supported by large-scale studies.

\section{References}

1. Hinman F, Gibson TE. Tumors of the epididymis, spermatic cord and testicular tunics: a review of the literature and report of three new cases. Arch Surg 1924;8(1):100-137

2. Bhosale PR, Patnana M, Viswanathan C, Szklaruk J. The inguinal canal: anatomy and imaging features of common and uncommon masses. Radiographics 2008;28(3):819-835

3. El Saadi AS, Al Wadan AH, Hamerna S. Approach to a giant inguinoscrotal hernia. Hernia 2005;9(3):277-279

4. Ahmed HU, Arya M, Muneer A, Mushtaq I, Sebire NJ. Testicular and paratesticular tumours in the prepubertal population. Lancet Oncol 2010;11(5):476-483

5. Hirano S, Kawaguchi S, Mikawa I, Motoi I, Masuda S. Giant hydrocele: two case reports. Hinyokika Kiyo 1991;37(2):195-198

6. Fletcher CD, Unni KK, Mertens F, eds. World Health Organization Classification of Tumours: Pathology and Genetics of Tumours of Soft Tissue and Bone. Lyon, France: IARC Press, 2002.

7. Sioletic S, Dal Cin P, Fletcher CDM, Hornick JL. Welldifferentiated and dedifferentiated liposarcomas with prominent myxoid stroma: analysis of 56 cases. Histopathology 2013; 62(2):287-293

8. Coleman J, Brennan MF, Alektiar K, Russo P. Adult spermatic cord sarcomas: management and results. Ann Surg Oncol 2003; 10(6):669-675 